\title{
Estrategias didácticas en escenarios de innovación tecnológica: Riquezas y complejidades pedagógicas Proyecto de Investigación \\ $\mathrm{N}^{\circ} 9.2$ \\ Equipo de Investigación ${ }^{(1)}$ \\ Cecilia Mazzeo
}

por Facultad de Diseño y Comunicación, Universidad de Palermo (ARG)

\begin{abstract}
Resumen: El Proyecto de Investigación 9.2 presenta y reflexiona, desde sus campos específicos de acción académica, sobre el impacto de la inclusión de las nuevas tecnologías digitales en el marco de la enseñanza de las disciplinas proyectuales, inclusión de recursos tecnológicos en los diferentes ámbitos de enseñanza y la práctica profesional, que ha impulsado el desarrollo de nuevas estrategias didácticas para la enseñanza del diseño. Las diversas condicionantes contextuales, que abarcan desde dimensiones económicas hasta tradiciones en la enseñanza del proyecto, han posibilitado el surgimiento de múltiples modos de inserción de la tecnología en las aulas y los talleres de diseño. Este variado panorama incluye desde proyectos de diseño mediados por tecnología, hasta estrategias de enseñanza y aprendizaje en escenarios virtuales y presenciales. Se propone instalar el debate sobre este amplio abanico de posibilidades.
\end{abstract}

Palabras clave: Diseño - Tecnología - Enseñanza - Innovación - Proyecto

[Resúmenes en inglés y portugués en la página 227]

${ }^{(1)}$ Los CVs del Equipo de Investigación pueden consultarse en el Capitulo Directores de Líneas y Coordinadores de Proyectos de esta misma Edición.

\section{Acerca del Proyecto 9.2}

Estrategias didácticas en escenarios de innovación tecnológica: Riquezas y complejidades pedagógicas

El Proyecto 9.2 Estrategias didácticas en escenarios de innovación tecnológica: Riquezas y complejidades pedagógicas, reflexiona de manera conjunta sobre las estratégicas de enseñanza de disciplinas proyectuales en escenarios de innovación tecnológica, con el objetivo de articular la mirada desde diferentes disciplinas y contextos académicos. 
Avanza en una investigación en la que participan académicos de distintas Instituciones, con la coordinación de Cecilia Mazzeo (UP), con el fin de generar un corpus sólido de estudio acerca de la enseñanza, en todos sus niveles y contextos como práctica compleja que involucra una multiplicidad de componentes y acciones, siendo que la Didáctica General la tiene como objeto de conocimiento y la Didáctica Proyectual complejiza su objeto desde la mirada disciplinar. Por esta razón, la inclusión de recursos tecnológicos en los diferentes ámbitos de enseñanza y la práctica profesional, ha impulsado el desarrollo de nuevas estrategias didácticas para la enseñanza del diseño. Las diversas condicionantes contextuales, que abarcan desde dimensiones económicas hasta tradiciones en la enseñanza del proyecto, han posibilitado el surgimiento de múltiples modos de inserción de la tecnología en las aulas y los talleres de diseño. Este variado panorama incluye desde proyectos de diseño mediados por tecnología, hasta estrategias de enseñanza y aprendizaje en escenarios virtuales.

Las reflexiones y los resultados obtenidos en el Proyecto 9.2 Estrategias didácticas en escenarios de innovación tecnológica: Riquezas y complejidades pedagógicas se continúan desarrollando en el Proyecto 9.3 Tradición e Innovación, Desafíos del Cambio Curricular: Debates, actores, contenidos y estrategias en el marco de la misma Línea de Investigación y bajo la misma Directora Cecilia Mazzeo.

Sus principales objetivos son:

- Aportar a la construcción de un corpus teórico específico para la enseñanza del Diseño.

- Instalar en el campo disciplinar un nuevo eje de producción teórica.

- Propiciar el intercambio de producciones teóricas interdisciplinares.

- Consolidar la producción teórica referida a la Didáctica Proyectual como campo específico de producción teórica.

- Compartir las diversas propuestas de enseñanza mediadas por tecnología, existentes en el campo de la enseñanza del Diseño en todas sus especialidades.

\section{Acerca de la Línea 9}

Enseñar Disciplinas Proyectuales dirigida por Cecilia Mazzeo, se desarrolla de manera ininterrumpida desde 2015 en la Facultad de Diseño y Comunicación (UP, Argentina), e incluye hasta el momento tres proyectos finalizados el 9.1 La dimensión ideológica de la enseñanza del Diseño y 9.2 Estrategias didácticas en escenarios de innovación tecnológica, coordinados por Cecilia Mazzeo (UP), y el 9.3 Tradición e Innovación, Desafíos del Cambio Curricular: Debates, actores, contenidos y estrategias, coordinado por Cecilia Mazzeo (UP) y Guillermo Sánchez Borrero (PUCE). 


\section{Mapa de Áreas y Proyectos}

El Proyecto 9.2 se vincula con todas las carreras de grado y posgrado correspondientes a la Facultad de Diseño y Comunicación.

\section{Productos y Resultados}

\section{a)- Publicaciones}

Cuaderno del Centro de Estudios de Diseño y Comunicación No84. (2020) Estrategias didácticas en escenarios de innovación tecnológica. Coordinación Cecilia Mazzeo. Año XXI, Octubre 2020, Buenos Aires, Argentina. ISSN: 1668-0227. Esta publicación documenta y comunica los resultados alcanzados en el proyecto de investigación 9.2 Estrategias didácticas en escenarios de innovación tecnológica, y a continuación se detallan los autores y artículos contenidos en ella:

Mazzeo, Cecilia (2020) Prólogo (Pp. 11 a 13)

Alberdi, Isabel (2020) Buceando en lo profundo. Metodología en el proceso de diseño gráfico. Apuntes sobre estrategias para abordar la enseñanza de la etapa de relevamiento (Pp. 15 a 29)

Anarella, Luciana (2020) Los medios digitales y la autogestión de saberes. Una experiencia pedagógica en la enseñanza del diseño (Pp. 31 a 45)

Chavez Mosquera, Gabriela (2020) El pulgarcito educado (Pp. 47 a 51)

Coppo, Alicia (2020) Estrategias de enseñanza del diseño para una nueva generación. El rol docente y el vínculo con el estudiante en el marco de las TIC'S (Pp. 53 a 67)

Dalle, Leandro (2020) Taller-mediante. Reflexiones críticas sobre una experiencia de amplificación del taller de diseño al medio virtual/digital (Pp. 69 a 90)

Mazzeo, Cecilia (2020) Renovaciones y persistencias. El taller y las tecnologías digitales (Pp. 91 a 106)

Muñoz, Patricia (2020) Incorporación de nuevos contenidos a la enseñanza desde la investigación (Pp. 107 a 128)

Sánchez Borrero, Guillermo (2020) La enseñanza del diseño a través del Diseño Social y las nuevas tecnologías (Pp. 129 a 146)

Actas de Diseño No30 (2020) XIV Encuentro Latinoamericano de Diseño "Diseño en Palermo" X Congreso Latinoamericano de Enseñanza del Diseño Comunicaciones Académicas. Semana Internacional del Diseño en Palermo. Enero 2020, Buenos Aires, Argentina. En esta publicación se documentan las ponencias de la Comisión Enseñar disciplinas proyectuales: Proyecto 9.2 Estrategias didácticas en escenarios de innovación tecnológica correspondientes a la presentación del Cuaderno 84 durante el IV Coloquio de Investigación y Desarrollo en Diseño (Ponencias en Pp. 48-49). 
Actas de Diseño No27 (2019 XIII Encuentro Latinoamericano de Diseño “Diseño en Palermo" IX Congreso Latinoamericano de Enseñanza del Diseño Comunicaciones Académicas. Marzo 2019, Buenos Aires, Argentina. En esta publicación se documentan las ponencias de la Comisión Enseñar disciplinas proyectuales: Proyecto 9.2 Estrategias didácticas en escenarios de innovación tecnológica correspondientes a la presentación del Cuaderno 84 durante el III Coloquio de Investigación y Desarrollo en Diseño (Pp. 54-55).

\section{b)- Congresos / Coloquios / Plenarios}

$3^{\circ}$ Plenario de Directores de Investigación DC. 26, 27 y 29 de agosto de 2019. En este tercer plenario los Directores de las Líneas y Proyectos de Investigación presentaron a sus pares y al conjunto del Programa de Investigación de la Facultad de Diseño y Comunicación, los resultados obtenidos y/o en proceso (publicaciones y acuerdos), junto con los avances de Proyectos. La Directora de la Línea de Investigación №9 presentó a sus pares, los resultados del proyecto 9.2 Estrategias didácticas en escenarios de innovación tecnológica, y anticipó las gestiones y vínculos en tratamiento para un futuro proyecto de la Línea.

IV Coloquio de Investigación y Desarrollo en Diseño Latino. Universidad de Palermo, 29 de julio de 2019. X Congreso Latinoamericano de Enseñanza del Diseño. Semana Internacional del Diseño en Palermo.

En la comisión Enseñar disciplinas proyectuales se presentaron las conclusiones del Proyecto 9.2 Estrategias didácticas en escenarios de innovación tecnológica, cuyas reflexiones continúan las iniciadas con el proyecto 9.1 La dimensión ideológica de la enseñanza del Diseño. La comisión se centró sobre las implicancias de la didáctica general, y la didáctica proyectual, la inclusión de recursos tecnológicos en la enseñanza y la práctica profesional y el desarrollo de nuevas estrategias didácticas en la enseñanza del diseño: desde proyectos de diseño mediados por tecnología, hasta estrategias de enseñanza y aprendizaje en escenarios virtuales. Expusieron: Cecilia Mazzeo, Isabel Alberdi, Luciana Anarella y Guillermo Sánchez Borrero.

A continuación se detallan las ponencias presentadas en esta Comisión coordinada por la Directora del proyecto Cecilia Mazzeo:

Cecilia Mazzeo (Argentina) - Coordinadora de la Comisión

Renovaciones y persistencias: El taller y las tecnologías digitales

Isabel Alberdi (Argentina)

Buceando en lo profundo: Metodología en el proceso de diseño gráfico. Apuntes sobre estrategias para abordar la enseñanza de la etapa de relevamiento

Luciana Anarella (Argentina)

Los medios digitales y la autogestión de saberes. Una experiencia pedagógica en la enseñanza del diseño

Leandro Dalle (Argentina)

Taller-mediante: Reflexiones críticas sobre una experiencia de amplificación del taller de diseño al medio virtual-digital 
Guillermo Sánchez Borrero (Ecuador)

La enseñanza del diseño a través del diseño Social y las nuevas tecnologías

$2^{\circ}$ Plenario de Directores de Investigación DC, 27 de agosto y 4 de septiembre - 2018. En este segundo plenario los Directores de las Líneas y Proyectos de Investigación presentaron a sus pares y al conjunto del Programa de Investigación de la Facultad de Diseño y Comunicación, los resultados obtenidos y/o en proceso (publicaciones y acuerdos), junto con los avances de Proyectos. La Directora de la Línea de Investigación No9 presento a sus pares, los resultados del Proyecto 9.1. La dimensión ideológica de la enseñanza del Diseño y los avances de su segundo proyecto 9.2 Estrategias didácticas en escenarios de innovación tecnológica.

III Coloquio de Investigación y Desarrollo en Diseño Latino. Universidad de Palermo, 30 de julio de 2018. IX Congreso Latinoamericano de Enseñanza del Diseño. Semana Internacional del Diseño en Palermo.

En la comisión Enseñar disciplinas proyectuales se presentaron las conclusiones del proyecto 9.1. La dimensión ideológica de la enseñanza del Diseño y los avances del 9.2 Estrategias didácticas en escenarios de innovación tecnológica, expusieron: Cecilia Mazzeo, María Isabel Alberdi y Leandro Dalle.

A continuación se detallan las ponencias presentadas en esta Comisión coordinada por la Directora del proyecto Cecilia Mazzeo:

Alberdi, Maria Isabel

Buceando en lo profundo. Metodología en el proceso de diseño gráfico. Apuntes sobre estrategias para abordar la enseñanza de la etapa de relevamiento

Dalle, Leandro

Taller-mediante. Reflexiones críticas sobre una experiencia de amplificación del taller de diseño al medio virtual-digital

Mazzeo, Cecilia

Renovaciones y persistencias. El taller y las tecnologías digitales

\section{c)- Formación de Posgrado e Impacto curricular}

La Directora Cecilia Mazzeo es Docente de Posgrado en Diseño de la Facultad de Diseño y Comunicación en Maestría en Gestión del Diseño y Doctorado en Diseño e incorpora los contenidos de su investigación a sus asignaturas en posgrado.

Abstract: Research Project 9.2 presents and reflects, from its specific fields of academic action, on the impact of the inclusion of new digital technologies in the teaching of project disciplines, inclusion of technological resources in the different teaching fields and professional practice, which has promoted the development of new didactic strategies 
for teaching design. The various contextual conditioning factors, ranging from economic dimensions to traditions in the teaching of the project, have made possible the emergence of multiple ways of inserting technology in classrooms and design workshops. This varied panorama includes everything from technology-mediated design projects to teaching and learning strategies in virtual and face-to-face scenarios. It is proposed to install the debate on this wide range of possibilities.

Keywords: Design - Technology - Teaching - Innovation - Project

Resumo: O Projeto de Pesquisa 9.2 apresenta e reflete, a partir de seus campos específicos de atuação acadêmica, sobre o impacto da inclusão de novas tecnologias digitais no ensino de disciplinas de projetos, inclusão de recursos tecnológicos nas diferentes áreas de ensino e prática profissional, que tem promovido o desenvolvimento de novas estratégias didáticas para o ensino de design. Os diversos condicionantes contextuais, que vão desde as dimensões econômicas às tradições no ensino do projeto, têm possibilitado o surgimento de múltiplas formas de inserção da tecnologia nas salas de aula e nas oficinas de design. Esse panorama variado inclui desde projetos de design mediados por tecnologia a estratégias de ensino e aprendizagem em cenários virtuais e presenciais. Propõe-se instalar o debate sobre este amplo leque de possibilidades.

Palavras chave: Design - Tecnologia - Ensino - Inovação - Projeto

[Las traducciones de los resúmenes fueron realizadas a través de traductor automático] 